\title{
Acoustical Excitation for Damping Estimation in Rotating Machinery
}

\author{
Bram Vervisch $^{a, b}$, Michael Monte $^{a, b}$, Kurt Stockman $^{a, b}$, Mia Loccufier $^{b}$ \\ ${ }^{a}$ Department of Electromechanical Engineering, Technical University of West-Flanders, Graaf Karel \\ de Goedelaan 5, B-8500 Kortrijk, Belgium \\ ${ }^{b}$ Departement of Electrical Engineering, Systems and Automation, University of Ghent, \\ Technologiepark 914, B-9052, Zwijnaarde, Belgium
}

\begin{abstract}
In experimental modal analysis a structure is excited with a force in order to estimate the frequency response function. Typically, this force is generated by a shaker or a hammer impact. Both methods have proven their usefulness, but have some well-known disadvantages. A main disadvantage of the shaker is that it has to be fixed to the structure whereas with a hammer it is not possible to excite a specific frequency. To overcome these disadvantages, alternative non-contact methods can be used. There are several non-contact techniques, i.e. pressurized air, laser, acoustics, etc. By using acoustics as an excitation technique it is easy to select an excitation signal going from random noise to a simple sine. Also the equipment to produce the acoustic excitation is rather cheap. However, the state of the art does not offer a straightforward technique to estimate the excitation force, making it difficult for applications such as experimental modal analysis. In this research, acoustic excitation is compared with hammer excitation to estimate the frequency response function of two shafts. Especially a method to validate the force induced by the acoustics is derived. The final purpose of this research is to estimate the damping properties of rotating machinery.
\end{abstract}

\section{Introduction}

In rotating machinery, damping plays an important role in the stability analysis [1]. If the bearings do not provide sufficient damping, a rotor can become unstable due to the presence of its internal damping, because the latter can excite instead of dissipate vibrational energy [2]. Several theoretical models have been investigated to estimate the stability threshold speed above which no stable operation is possible [3][4][5]. In order to validate these models, experimental methods are necessary. Non-contact excitation and measurement techniques are required because the system is rotating.

Excitation with an electrodynamic shaker has proven its usefulness in experimental modal analysis. With a shaker, several excitation signals such as random noise or simple sines are possible. Furthermore, the excitation force and the acceleration can be measured accurately by using an impedance head [6]. The shaker can also be used on a rotating shaft by applying the force on an existing or a dedicated bearing [7]. This bearing, however, not only introduces mass loading, but also adds extra damping, making it inefficient for accurate damping estimation. With a hammer impact, this drawback can be avoided. Nevertheless, there are also some significant disadvantages when using this technique [8]. For one thing, repeatability is a concern that becomes even a bigger problem when the system is rotating. Also, there is still the possibility of damaging the system and only one type of excitation, namely impact, is possible.

Therefore, non-contact excitation techniques become important. These techniques are mainly pressurized air [9][10], laser [11], and acoustics [12]. Laser excitation can only be used if very small forces are needed. Pressurized air can produce higher forces, but has a limited bandwidth due to by the minimum pulse duration. With acoustic excitation, the forces are also small, but high enough to get a measurable displacement. Moreover, any kind of excitation signal can be used. However, it is difficult to measure the excitation force caused by the acoustic pressure. Therefore, acoustic excitation is used mostly in operational modal analysis, where the excitation force does not have to be known [13],[12]. Nevertheless, if the goal is to estimate the damping accurately, it is preferable to know the excitation force. In this research an effort is made to estimate the force caused by the acoustic excitation.

In section 2, the acoustic excitation is explained for the application of shaft excitation. Subsequently, in section 3 , acoustic excitation is used to measure one frequency response function, or FRF, of two non-rotating shafts: one shaft supported by two wires in the nodes of the first free mode approximating a free-free beam and a second shaft on rolling element bearings. Section 4 proposes a calibration procedure for the estimation of the force produced by the acoustic pressure. 


\section{Acoustic excitation}

Acoustic excitation is a technique that has some specific advantages in comparison with traditional techniques such as hammer and shaker excitation. Besides being non-contact, the choice of the excitation signal is arbitrary making parameters such as bandwidth easy to deal with. In Table 1 some of the main characteristics of traditional and newer techniques are summed up.

Table 1 - Comparison of excitation techniques

\begin{tabular}{llcll}
\hline Excitation technique & Contact/non-contact & Force range [N] & Excitation signal & Repeatability \\
\hline Elektrodynamic shaker & contact & $8-1800$ & arbitrary & fair \\
Hammer & contact & $5-45000$ & impact & poor \\
Laser & non-contact & $<5 \times 10^{-4}$ & arbitrary & fair \\
Pressurized air & non-contact & $<0,6$ & impact & fair \\
Acoustics & non-contact & $<5 \times 10^{-2}$ & arbitrary & fair \\
\hline
\end{tabular}

\subsection{Force by acoustic excitation}

In this research acoustic excitation is used to excite a cylindrical shaft with a length-diameter ratio of 100 . Because the shaft is chosen to be thin, small excitation forces will result in a measurable displacement. While hammer or shaker excitation can be assumed to be single-point, acoustic pressure produces multiple-point excitation. Nevertheless, it can be shown that modal parameter estimation is similar [8]. The forces acting on the shaft can be calculated by integrating the acoustic pressure over the surface exposed to the pressure. However, the calculation of the acoustic pressure is far from straightforward and can be calculated with an exact knowledge of the source (sound power, directivity,...) by using numerical techniques [14]. Without this knowledge, the state of the art does not provide direct technique to calculate the force. Here, a simplified estimation of the force is made, by measuring the pressure at a certain distance and assuming that this pressure is equal on the whole surface of the shaft.

The source is an Atlas Sound PD60A compression driver. These kind of drivers are used in multiple purpose high power horns. In Table 2, the specifications taken from the datasheet are shown. The construction of the driver (Figure 2) allows the assumption of plane wave propagation if considered near to the source. Therefore, the shaft will always be placed at a two millimeter distance, in the near field, for low frequencies. At this distance, the force equals the measured sound pressure multiplied by the exposed surface:

$$
F_{\text {acoustic }}=P_{\text {acoustic }} \frac{\pi d l_{\text {exposed }}}{2}
$$

with $P_{\text {acoustic }}$ the pressure measured at two millimeter from the source, $d$ the shaft diameter and $l_{\text {exposed }}$ the length of the shaft part exposed to the pressure. This last parameter, by assuming plane wave propagation, equals the diameter of the tube where the sound leaves the source. The amplifier is a Saigor 100, a regular consumer amplifier. In order to get the same pressure in the different measurements, the amplification is kept the same during the whole experiment.

\subsection{Excitation signal}

With acoustical excitation, different excitation signals are possible. Multi-sine, random noise and even pulses can be imposed to the system. However, by having a relatively small acoustic force, all combined signals divide the force over the frequency band. This can be seen in Figure $1 \mathrm{a}$ and $1 \mathrm{~b}$ where the sound pressure is shown for Gaussian white noise in the time domain and frequency domain. To use the acoustic source at full power, a stepped sine is imposed. While this method is time consuming, it leads to accurate results. 
Table 2 - Specifications of the Atlas Sound PD60A taken from the datasheet

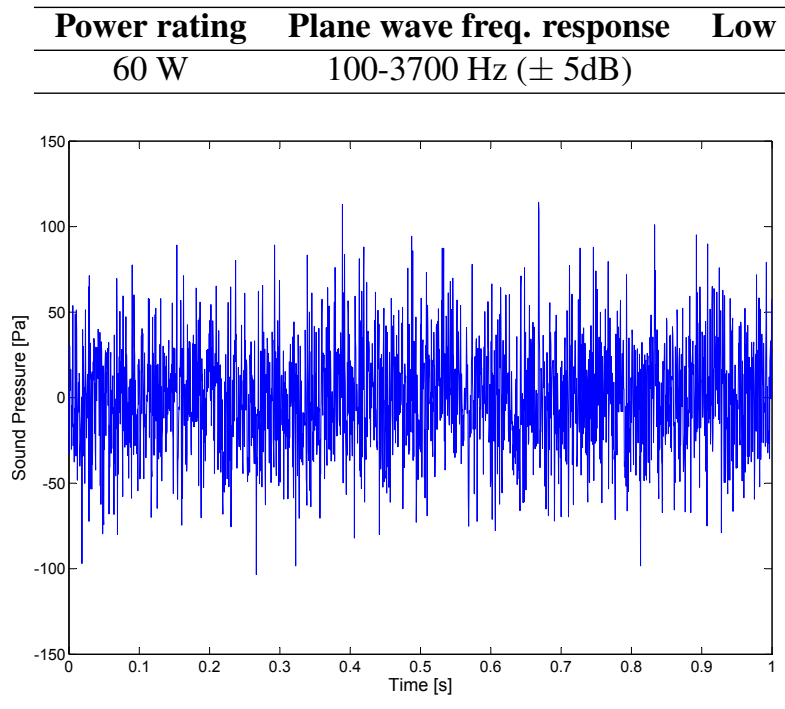

(a) Sound pressure in time domain

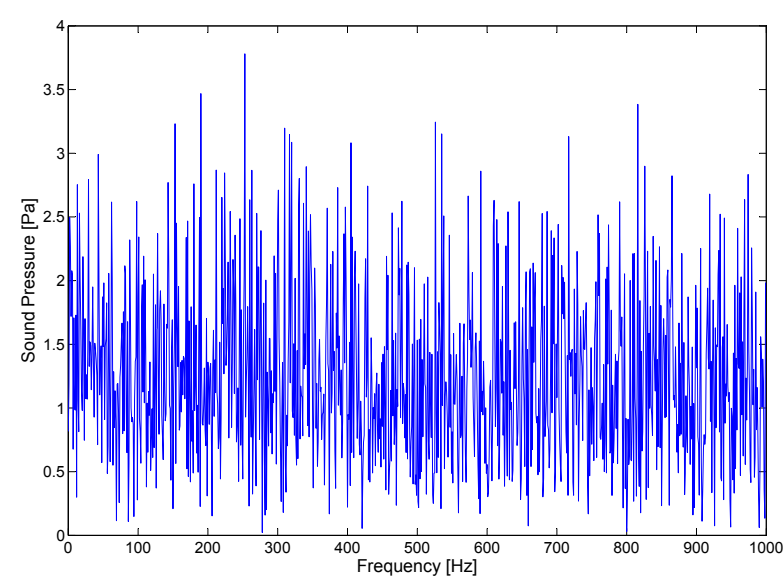

(b) Sound pressure in frequency domain

Fig. 1 - Sound Pressure of Gaussian white noise with a sample frequency of $2000 \mathrm{~Hz}$ in time and frequency domain

\section{Shaft experiments}

\subsection{Sound pressure of the source}

The pressure of the source is measured with a microphone PCB 130D20 in front of the source. The amplifier level is calibrated in a way that the pressure has an amplitude of $54.27 \mathrm{~Pa}$ for a $400 \mathrm{~Hz}$ sine. Because the source can produce the highest power in an 400 to $500 \mathrm{~Hz}$ range (Figure 5), the pressure is measured from $400 \mathrm{to} 450 \mathrm{~Hz}$, with a resolution of $1 \mathrm{~Hz}$. The results are depicted in Figure 3. It can be seen that there is a slight rise in the pressure when the frequency increases, which also corresponds to the trend in Figure 5.

\subsection{Free-free shaft}

As a proof of concept, the acoustic excitation technique is applied on a shaft in a free-free configuration. This configuration is accomplished by suspending the shaft on two wires in the nodes of the first free mode. The FRF is measured while exciting and measuring at the same location, $0.425 \mathrm{~m}$ from the left side. The shaft is in steel CF53 with a density of 7730 $\mathrm{kg} / \mathrm{m}^{3}$ and a Young modulus of $2.2 \times 10^{11} \mathrm{~Pa}$. The diameter is $0.010 \mathrm{~m}$ and the length $1.00 \mathrm{~m}$. At first the shaft is excited by a hammer to get a reference FRF. Secondly the shaft is excited with the sound source at exactly the same place (Figure 4). The displacement is measured by using eddy current probes in order to eliminate the mass-loading of the sensors.

By assuming that the force can be calculated by (1) and taking the pressures of Figure 3, the frequency response of the shaft can be calculated directly from displacement measurements. The comparison between the hammer excitation and the acoustic excitation is shown in Figure 9a. For the hammer excitation, no window is used on the measurements, to avoid amplitude loss. Also, the FRF of the hammer impact is the result of averaging five FRF's. Both FRF's agree quite well, even with the simplified force estimation. Moreover, if the damping factor of the shaft is calculated with a simple peak-amplitude method [15], both damping factors are the same, i.e. 0.0013. The resonance frequency is $423 \mathrm{~Hz}$ and corresponds to the fourth free mode. It deviates slightly from the theoretical expected value of $411 \mathrm{~Hz}$.

\subsection{Shaft on bearings}

The same technique as in the free-free shaft is used for a shaft on bearings (Figure 4 and 7). The shaft is also in steel CF53 with a density of $7730 \mathrm{~kg} / \mathrm{m}^{3}$ and a Young modulus of $2.2 \times 10^{11} \mathrm{~Pa}$. The diameter is $0.010 \mathrm{~m}$ and the length $1.14 \mathrm{~m}$. The shaft is placed on angular contact high precision bearings. Although clamped boundary conditions are intended, a result inbetween clamping and supporting is obtained. Also, it should be noted that the stiffness properties of this configuration 


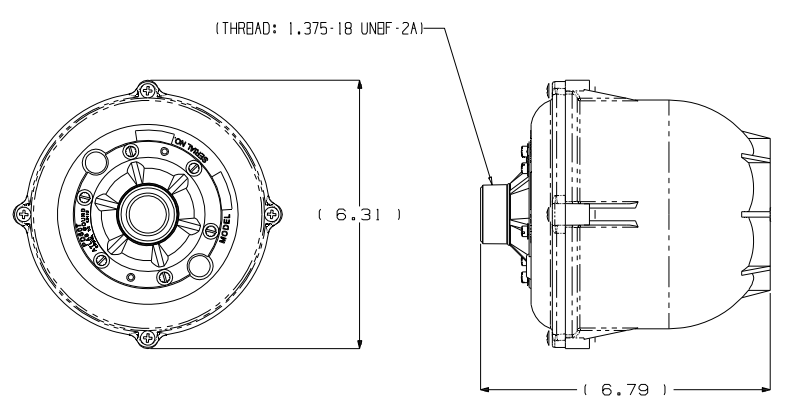

Fig. 2 - Drawing of the Atlas Sound PD60A (dimensions in inch)

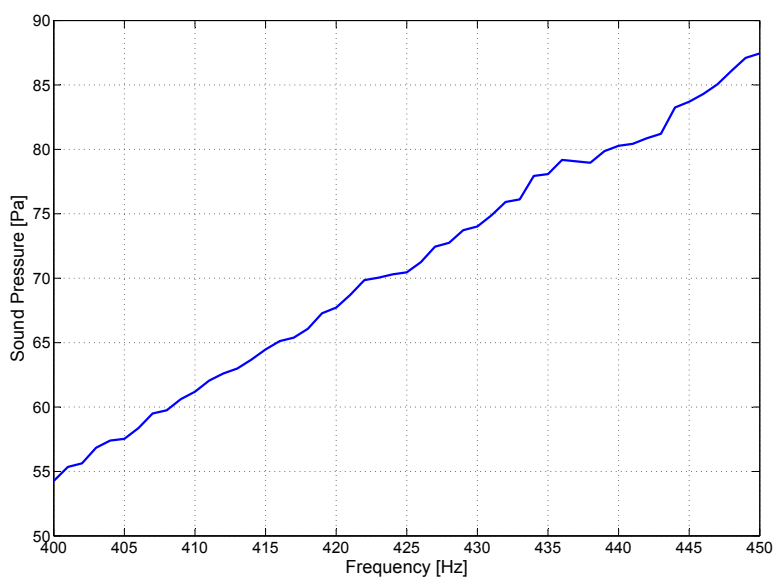

Fig. 3 - Sound pressure of the source measured at a distance of $2 \mathrm{~mm}$ for a fixed setting of the amplifier

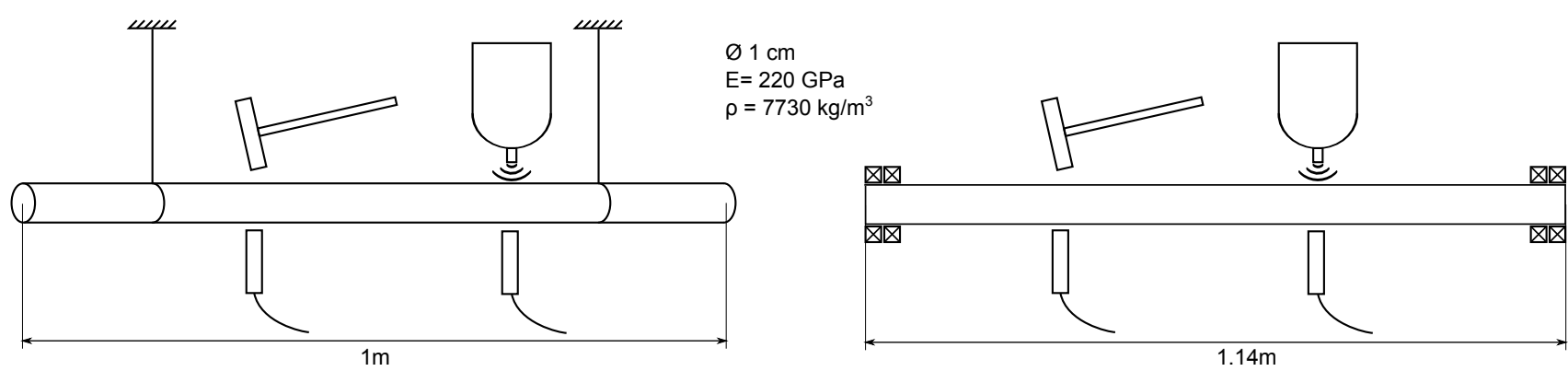

Fig. 4 - Impact and acoustic excitation of a free-free shaft and a shaft on bearings 


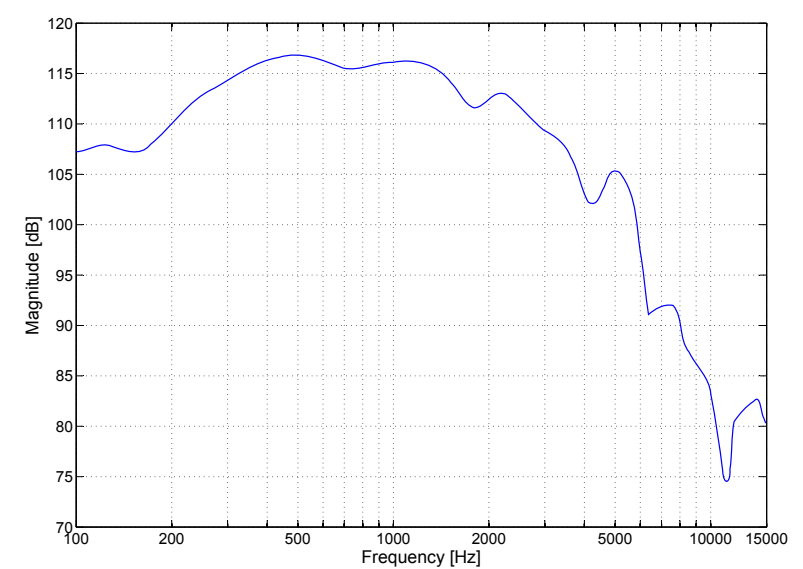

Fig. 5 - PD-60A Plane Wave Tube Response taken from the datasheet

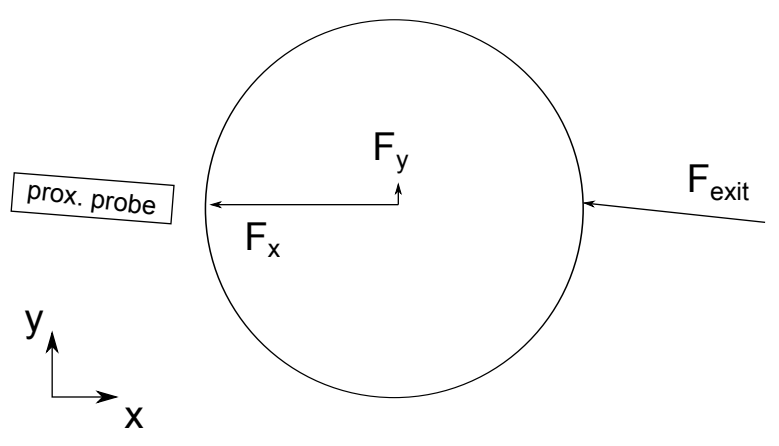

Fig. 6 - Deviation of the excitation force and displacement measurements leading two double resonance frequencies

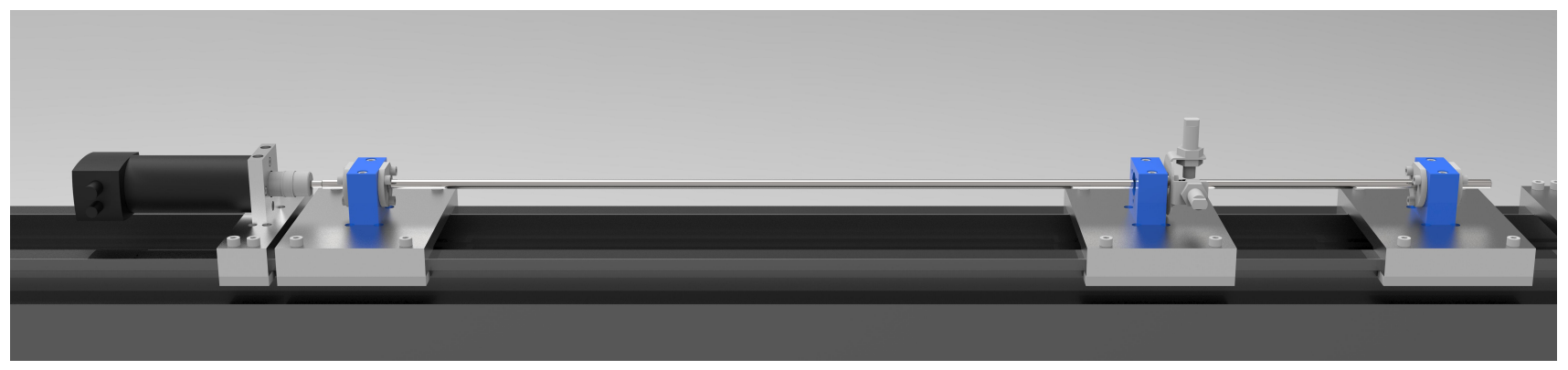

Fig. 7 - Drawing of the shaft on bearings

are not exactly the same for both directions in a chosen $x-y$ cordinate system and the shape of the shaft surface implies excitation in both directions, as illustrated in Figure 6. Because the proximity probe can never be put exactly in one direction, both movements are measured. Two resonance frequencies close to each other can be expected. To validate this phenomenon, a measurement is made with hammer excitation and proximity probes in both directions and exciting in the x-direction. In Figure 8a, both displacements, filtered with a bandpass filter between 400 and $450 \mathrm{~Hz}$, are plotted. Both displacements start in phase but have a slightly different frequency. This results in a trajectory depicted in Figure $8 \mathrm{~b}$. In this trajectory it is clear that the shaft does not only move in the $x$-direction, but has a small movement in the $y$-direction too.

For a clamped shaft, a resonance frequency of $483 \mathrm{~Hz}$, corresponding to the fifth mode, is theoretically calculated. Because ideal clamping is not accomplished, a lower frequency can be expected. As can be seen in Figure 9b, the FRF meets the expectation. The FRF measured with hammer impact indicates that there are two resonance frequencies close to each other, i.e. 424 and $439 \mathrm{~Hz}$. The one with acoustic excitation is slightly different, having two resonance frequencies of 423 and $439 \mathrm{~Hz}$. By using the peak-amplitude method on these measurements, the hammer impact technique leads to respectively a damping factor of 0.0040 and 0.0035 and the acoustic excitation leads to both 0.0045 . These differences should be interpreted carefully. Firstly, the force is estimated with a simplified method. Secondly, the frequency resolution is only $1 \mathrm{~Hz}$, resulting in an error on the absolute peak, i.e. picket fence effect [16]. Thirdly, and most importantly, with the hammer impact technique it is not possible to hit the shaft every time on exactly the same place. This means that, the five separate FRF's that are measured and averaged, can deviate depending on the hit. With acoustic excitation, the position of the source with respect to the shaft does not change. Therefore, acoustic excitation assures repeatability.

\section{Calibration procedure}

Because of the difficulty to estimate the excitation force theoretically, a calibration procedure is proposed, to increase the accuracy of the results. Figure 10 shows the test setup. A sound source and a shaft sample are mounted on a variable 


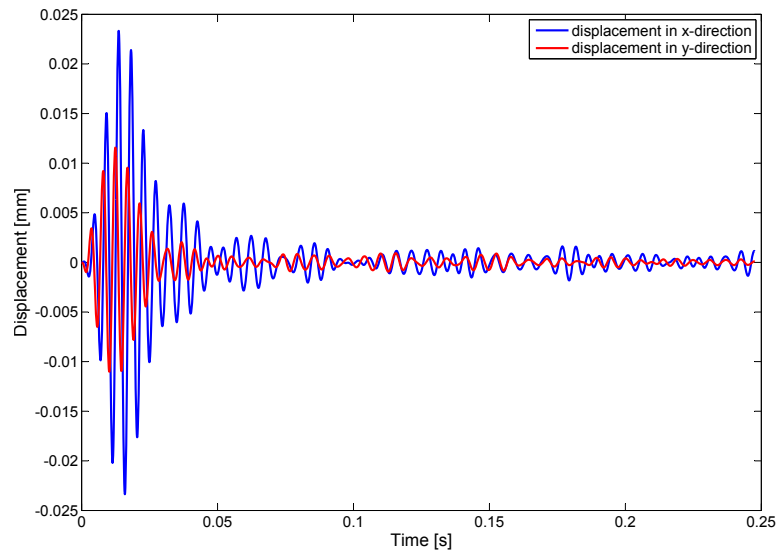

(a) Displacement as a function of time

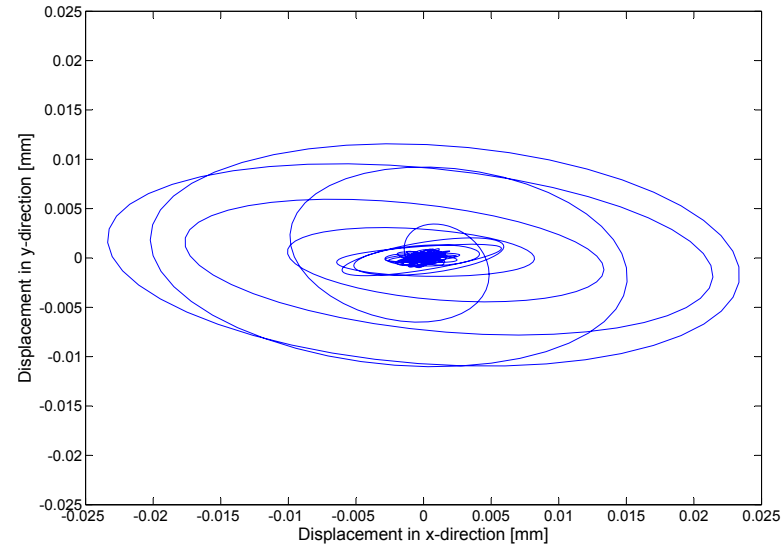

(b) Trajectory of displacements

Fig. 8 - Displacement of the shaft with hammer excitation for frequencies between 400 and $450 \mathrm{~Hz}$

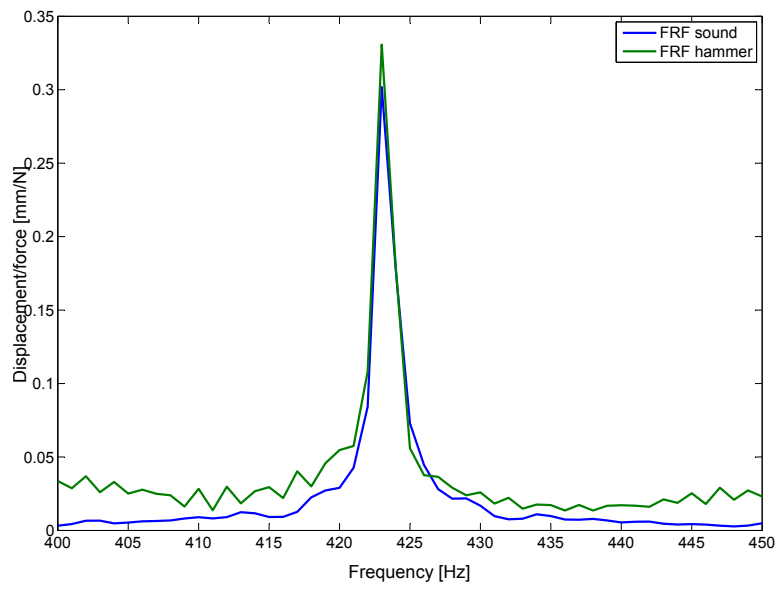

(a) FRF's of the free shaft

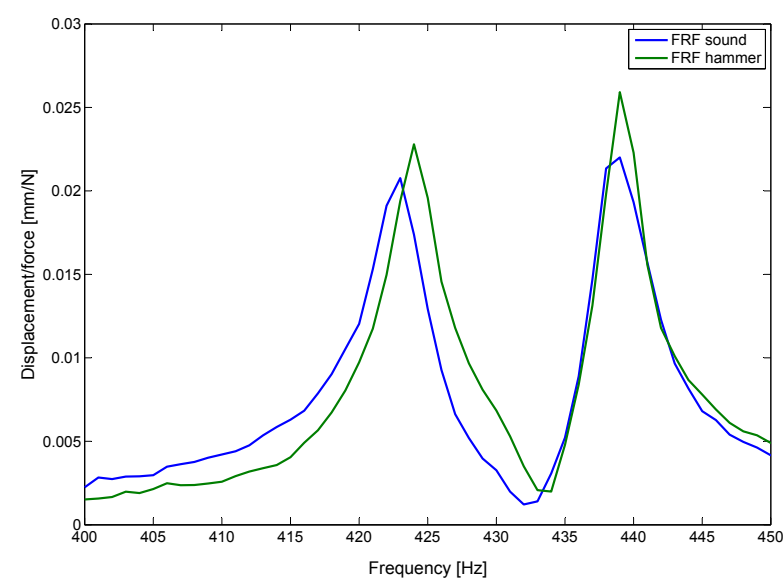

(b) FRF's of the shaft on bearings

Fig. 9 - FRF's with acoustic vs. hammer excitation for frequencies between 400 and $450 \mathrm{~Hz}$ 


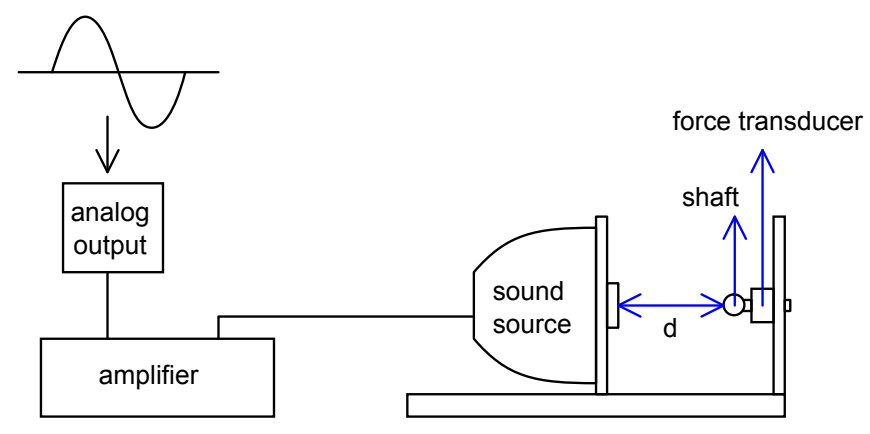

Fig. 10 - Setup for calibration procedure

distance. The samples are bolted to a force transducer. The sample shafts have a varying length so that the influence of the force on the length of the shaft can be verified. If the length of these samples is small, they can be assumed to be rigid for low frequencies. A thorough testing set which will yield calibration charts will be included in future work.

\section{Conclusions}

Acoustic excitation is a non-contact excitation technique that can be used to measure frequency response functions of shafts. By using this technique, the shaft is excited by a force which equals the pressure integrated over the surface of the shaft. Different kinds of excitation signals can be used. However, if multi-sine signals are used, the force is divided over the different frequencies, resulting in a lower force per frequency. In comparison to hammer impact, acoustic excitation leads to an exactly reproducible force, while hammer impact depends on the hit. In this research a simplified estimation of the excitation force is made by multiplying the acoustic pressure, measured by a microphone, with the excited surface. The method is validated by measuring a frequency response function of a shaft supported by two wires at the nodes of the first free mode and a shaft on bearings and comparing it to the hammer impact. For both shafts, the method leads to acceptable results, and gives similar damping factors when calculated with a peak-amplitude method. However, if an exact damping measurement is necessary, the simplified force estimation will not suffice. Therefore, a calibration procedure is proposed. Furthermore, the method has some practical drawbacks. By using the stepped-sine, it is rather time-consuming compared to the hammer impact. On top of that, the high acoustic pressure require the executor to wear hearing protection. Hence, Acoustic excitation is advisable when non-contact excitation is required, for example in rotordynamics and when effects of mass loading and other influences are of a particular concern. In future work, besides the calibration procedure, numerical techniques can be used to estimate the pressure of the source. Also, other sources, with a better frequency response for lower frequencies can be used. If higher power can be accomplished, combined signals such as noise can be evaluated.

\section{References}

[1] B Vervisch, K Stockman, and M Loccufier. Sensitivity of the stability threshold in linearized rotordynamics. ISMA conference, 2012.

[2] Mohamed A Kandil. On Rotor Internal Damping Instability. PhD thesis, 2004.

[3] L Forrai. Instability due to internal damping of symmetrical rotor-bearing systems. JCAM, 1(2):137-147, 2000.

[4] L Forrai. A finite element model for stability analysis of symmetrical rotor systems with internal damping. JCAM, 1(1):37-47, 2000.

[5] Giancarlo Genta and Nicola Amati. Hysteretic damping in rotordynamics: An equivalent formulation. Journal of Sound and Vibration, 329(22):4772-4784, October 2010.

[6] MA Peres, RW Bono, and DL Brown. Practical Aspects of Shaker Measurements for Modal Testing. processvibration.com, pages 2539-2550.

[7] I. Bucher and D. J. Ewins. Modal analysis and testing of rotating structures. Philosophical Transactions of the Royal Society A: Mathematical, Physical and Engineering Sciences, 359(1778):61-96, January 2001. 
[8] Jinzhi Wu and FA Moslehy. On modal testing using speaker for excitation. IMAC-XIII proceedings, (7):24-29, 1995.

[9] S. Vanlanduit, F. Daerden, and P. Guillaume. Experimental modal testing using pressurized air excitation. Journal of Sound and Vibration, 299(1-2):83-98, January 2007.

[10] R. Farshidi, D. Trieu, S.S. Park, and T. Freiheit. Non-contact experimental modal analysis using air excitation and a microphone array. Measurement, 43(6):755-765, July 2010.

[11] P Castellini. Measurement of vibrational modal parameters using laser pulse excitation techniques. Measurement, 35(2):163-179, March 2004.

[12] Y F Xu and W D Zhu. Rotating Machinery, Structural Health Monitoring, Shock and Vibration, Volume 5. 5:359-374, 2011.

[13] H Herlufsen and N Mø ller. Operational Modal Analysis of a Wind Turbine Wing using Acoustical Excitation. System, (1):1-8.

[14] Frank J. Fahy. Sound and Structural Vibration: Radiation, Transmission and Response. Academic Press, 1987.

[15] D. J. Ewins. Modal Testing: Theory, Practice and Application (Mechanical Engineering Research Studies: Engineering Dynamics Series). Wiley, 2001.

[16] R. B. Randall. Frequency Analysis. Bruel \& Kjaer, 1987. 